\title{
Flow Field in the Turbine Rotor Passage in an Automotive Torque Converter Based on the High Frequency Response Rotating Five-hole Probe Measurement
}

\author{
Part I: Flow Field at the Design Condition (Speed Ratio 0.6)
}

\author{
Y. F. LIU, B. LAKSHMINARAYANA* and J. BURNINGHAM
}

Center for Gas Turbines and Power, The Pennsylvania State University, 153 Hammond Building, University Park, PA 16802

(Received 11 May 2000; In final form 23 May 2000)

\begin{abstract}
The relative flow field in an automotive torque converter turbine was measured at three locations inside the passage (turbine $1 / 4$ chord, mid-chord, and $4 / 4$ chord) using a highfrequency response rotating five-hole-probe. "Jet-Wake" flow structure was found in the turbine passage. Possible flow separation region was observed at the core/suction side at the turbine $1 / 4$ chord and near the suction side at the turbine mid-chord. The mass averaged stagnation pressure drop is almost evenly distributed along the turbine flow path at the design condition $(S R=0.6)$. The pressure drop due to centrifugal and Coriolis forces is found to be appreciable. The rotary stagnation pressure distribution indicates that there are higher losses at the first half of the turbine passage than at the second half. The major reasons for these higher losses and inefficiency are possible flow separation and a mismatch between the pump exit and the turbine inlet flow field. The fuel economy of a torque converter can be improved through redesign of the core region and by properly matching the pump and the turbine. The Part I of the paper deals with the design speed ratio $(S R=0.6)$, and Part II deals with the off-design condition $(S R=0.065)$ and the effects of speed ratio.
\end{abstract}

Keywords: Torque converter; Turbine; Flow measurement; Rotating frame of reference; Five-hole probe

\section{INTRODUCTION}

A major effort has been made in recent years to reduce the automobile fuel consumption and air pollution. One of the important components that influence the fuel economy of the automatic transmission car is the torque converter. Since several million torque converters are built every

\footnotetext{
*Corresponding author. Tel.: 814 865-5551, Fax: 814 865-7092, e-mail: bllaer@engr.psu.edu
} 
year, even a small improvement in efficiency would result in significant fuel savings. The objective of this research was to improve the torque converter performance through improved knowledge of the flow field.

A typical torque converter consists of a pump, a turbine, and a stator, and it employs oil as the working fluid. The pump is connected to the engine crankshaft and converts energy from the automobile engine to fluid power. In contrast, the turbine extracts this energy from the fluid to the transmission shaft. The stator acts as a reaction member, which is placed between the turbine exit and the pump inlet. Its function is to redirect the turbine exit flow back into the pump.

Some of the earlier investigations on torque converter are briefly described below. Browarzik (1994) used hot film to examine the unsteady flow field at the inlet and exit of a pump and turbine of a Fottinger type torque converter. Marathe et al. (1997) measured the turbine exit flow using a high frequency response five-hole probe. Later, Dong (1998) measured the pump and turbine exit flow using an improved miniature five-hole probe. By and Lakshminarayana (1995) used pressure taps to measure the static pressure in the pump and turbine. Brun and Flack (1997a, b) used a laser velocimeter to investigate flow in the pump and turbine blade passages. A review of the investigations carried out by two groups: the University of Virginia and the Pennsylvania State University, and an assessment of the fluid dynamics of an automotive torque converter is given by Von Backstrom and Lakshminarayana (1996).

None of the prior investigators, however, report detailed pressure and velocity field in the turbine rotor of a torque converter. The thrust of this paper is to bridge this gap. The novelty of this investigation is in the use of a rotating five-hole probe and the measurement of the flow field in the relative system.

Compared with the pump and stator flow, the turbine flow has many complex features. (1) The turbine passage has a large turning angle $\left(124^{\circ}\right)$; and due to this increased flow turning the curvature effect on the flow is large. (2) The turbine has a lower rotational speed than the pump, and the effects of the centrifugal and Coriolis force on the flow field in the turbine are smaller than that in the pump. Furthermore, the rotational effects vary with the speed ratio. At the near-stall condition, the turbine speed is almost zero, and the rotating effects are negligible. (3) The relative total velocity at the turbine inlet and the exit is relatively high due to large blade turning angle.

The pressure and velocity fields at three axial locations of the turbine (turbine $1 / 4$ chord, midchord, and 4/4 chord) are presented and interpreted in this paper. The investigation was carried out in a $245 \mathrm{~mm}$ torque converter using a miniature five-hole probe with high frequency response. For the sake of brevity, only the data at the design condition $(S R=0.6)$ are reported in this paper. The flow field at the off-design condition and effects of speed ratio are given in Part II of the paper.

\section{FACILITY AND ROTATING PROBE MEASUREMENT TECHNIQUE}

This experimental investigation was accomplished using the Torque Converter Research Facility at the Pennsylvania State University. The detailed description of this system can be found in Dong (1998). The pump has 32 blades, and the turbine has 36 blades. The inlet blade angle of turbine is $61.4^{\circ}$, and the outlet blade angle is $-62.5^{\circ}$. The flow field in the turbine passage was measured using a high-frequency response five-hole probe at five different speed ratios, 0.8, 0.6, 0.4, 0.2 and 0.065 . The accuracy of this rotating probe is the same as the probe used in the stationary frame of reference (Marathe, 1997); $\pm 0.15 \mathrm{~m} / \mathrm{s}$ in total velocity, $\pm 1.5^{\circ}$ in flow angles, and $\pm 700 \mathrm{~Pa}$ in pressure.

In order to simulate all the operating conditions of the torque converter, tests were conducted at five speed ratios: $0.8,0.6,0.4,0.2$ and 0.065 , 
TABLE I Test conditions and performance parameters

\begin{tabular}{lrrrrr}
\hline Speed ratio & \multicolumn{1}{c}{0.8} & \multicolumn{1}{c}{0.6} & \multicolumn{1}{c}{0.4} & \multicolumn{1}{c}{0.2} & 0.065 \\
\hline Pump rpm & 1100 & 1060 & 1090 & 1070 & 800 \\
Turbine rpm & 880 & 696 & 436 & 214 & 52 \\
Torque Ratio & 0.976 & 1.2590 & 1.507 & 1.771 & 1.922 \\
Efficiency \% & 78.08 & 75.54 & 60.28 & 35.41 & 12.49 \\
\hline
\end{tabular}

ranging from the peak efficiency condition $(S R=0.8)$ to the near stall condition $(S R=$ 0.065). The pump and turbine speeds for these speed ratios are listed in Table I. The performance data, torque ratio, and efficiency, measured by the load cell are also listed in the same table. The oil temperature inside the torque converter is controlled at $60^{\circ} \pm 1^{\circ}$.

Three cross sections are chosen for the study of turbine passage flow; turbine $1 / 4$ chord, midchord, and $4 / 4$ chord. The measurement planes are shown in Figure 1. The turbine $1 / 4$ chord location is mainly designed to assess the turbine inlet flow losses, the extent of flow separation, the pump blade wake decay, and so on. The mid-chord location is very important in understanding the dominant flow structure in the turbine passage to determine the major loss mechanism and flow

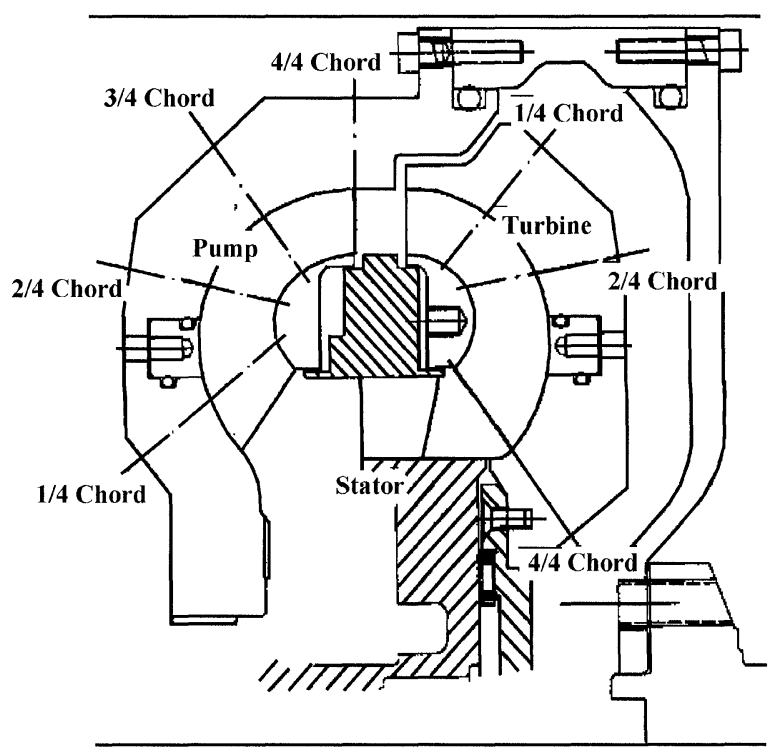

FIGURE 1 Cross sections of the troque converter used for rotating probe measurements. transport mechanism. The turbine $3 / 4$ chord is not suitable for measurement because of the large blockage effect of the probe. At the turbine $4 / 4$ chord, the stem of the probe is located outside the turbine passage, and the probe can travel radically straight, but not at the same angle as the turbine blade. Thus the probe blockage effect is largely reduced. The turbine $4 / 4$ chord flow field data is critical in determining the size of the core flow separation, the turbine passage periodic flow decay, and the flow mixing mechanism downstream of the turbine blade trailing edge.

The rotating probe measurement system designed, developed, and used in this research is described in Dong (1998). It consists of (1) a probe traversing mechanism to measure the flowfield at various chordwise and tangential locations, (2) a signal conditioner (amplifiers and battery) mounted on the rotating probe casing, (3) electrical conduits to transmit the data to slip-ring unit, and (4) a slip-ring unit. The probe design and data acquisition and processing systems in the stationary system are identical to those described earlier.

One of the most difficult problems encountered was in the design of the probe traverse system. The probe must be traversed both tangentially and radially relative to the blade passage. The probe is designed to be mounted on the pump shell at some specified location (percentage of the blade chord length, as shown in Fig. 1). The radial traverse of the probe is accomplished by a sliding sleeve, which has a series of index holes on its outer diameter. The sleeve can be translated (shell-tocore) in the pump shell and be fixed in incremental positions by a set screw, which engages the holes on the sleeve. The guide holes for the probe sleeve are manufactured at the specified locations on the pump shell and are aligned with the local blade angle. To carry out the tangential traverse, the blade system was designed to be rotated relative to the pump shell. The contour of shell is manufactured to match perfectly with the contour of the blade tip to keep the tip leakage flow negligible. The angular position of blade relative to the probe is determined by a group of index holes, which are 
accurately drilled by a $\mathrm{CNC}$ (Computer Numerical Control) machine. Once the probe tangential position is fixed, the shell and blade parts are held together by eight bolts. The pump cover is redesigned into two pieces; an annular ring and a flat plate cover. The electrical wires used to transfer the signal to the slip-ring unit are connected through two small holes in the pump cover from the pump shell to the pump shaft. All test parts mentioned above were manufactured by CNC machining, and the pump blade part was made by five-axis CNC milling.

The error in the rotating probe measurement is strongly dependent on the noise of the electronic system. The Kulite pressure transducers, which are used in the high-frequency response probe, require a very stable voltage DC power supply. The output signals of these transducers need to be amplified by low noise amplifiers. These amplifiers are powered by a DC power supply. For the rotating frame measurement, the signal has to be transferred from the rotating frame to the stationary frame, which is then processed through a data acquisition system (DAS-50 and 486 PC). The contribution to the electronic noise comes from the pressure transducer, the amplifier, the power supply, and the data acquisition system.

A slip-ring unit was used to transfer the electrical signal from the rotating frame to the stationary frame. This slip-ring unit is a customdesigned, high quality, brush-type unit. It has 37 channels, and the brushes and rings are made of gold alloy. At the shaft speed range from 0 to $1500 \mathrm{rpm}$, the noise level of this slip-ring unit is about 5 to $7 \mathrm{mV}$ for a 3 to $5 \mathrm{~V}$ level signal. This represents a signal noise ratio of $0.10 \%$ to $0.25 \%$. For the flow measurement, this noise level is acceptable after amplification, where the DC signal is about 3 to $5 \mathrm{~V}$ and the AC signal is about 20 to $50 \mathrm{mV}$. However, this noise is not acceptable before amplification, because it is even higher than the flow signal and will be amplified by the amplifier gain (250 in this system). Therefore, the amplifier and the transducer power supply have to be installed in the rotating frame before transmitting through the slip-ring unit.

A schematic of the electronic system of the rotating five-hole probe is shown in Figure 2. Two groups consisting of a rechargeable battery and a voltage regulator are used as the transducer power supply. The DC power supplies $(+12 \mathrm{~V},-12 \mathrm{~V})$ for the five amplifiers are transmitted through the slip-ring unit by five parallel channels each. Two voltage regulators are used in the rotating frame to reduce the noise of these power supplies due to the slip-ring unit. All the above electronic units and five signal amplifiers are custom-designed miniature parts. They are mounted on the pump shell, rotating with the pump passage. The output signal from each channel of the five-hole probe is transmitted to the stationary frame through four parallel channels of the slip ring unit to reduce the noise due to the slip-ring unit. Seven slip-ring channels were parallel connected to the common ground. The measured noise of this system is low, total about 5 to $6 \mathrm{mV}$, which is the same level as that measured in the stationary five-hole probe system. Since the probe is in the rotating frame, the transducers are not located at the same radius

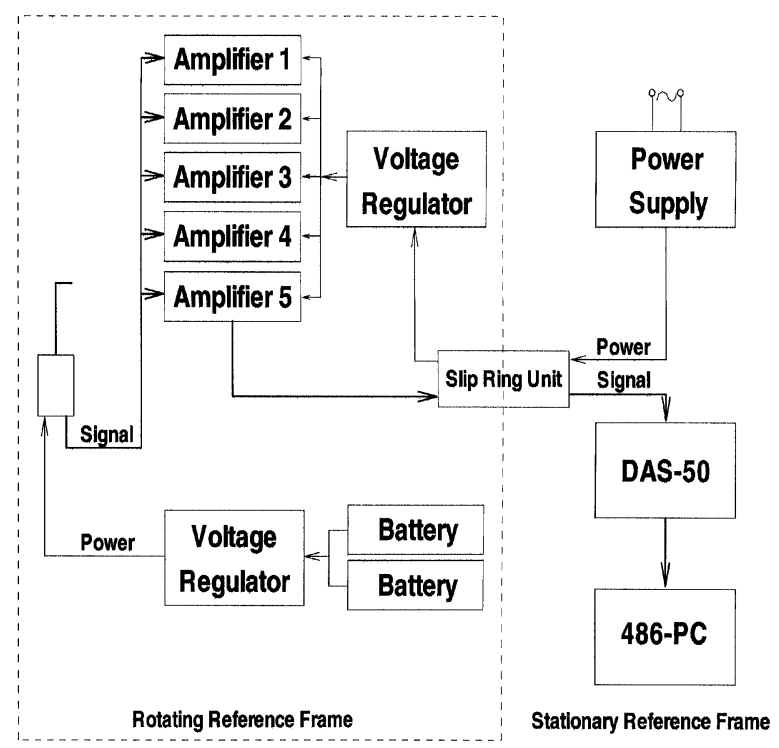

FIGURE 2 Schematic of electrical system for rotating probe measurements. 
as the tip of the probe, and all the measured pressures were corrected for the centrifugal force effect. The accuracy of the centrifugal force correction depends on the spatial error in measuring the location of the probe tip and the transducer sensors.

\section{DATA CAPTURE AND PROCESSING}

\section{Steady Flow Field Data Processing}

The velocities and pressures presented in this paper are normalized in the following way

$$
\begin{gathered}
W_{\text {norm }}=\frac{W}{V_{\text {ref }}}, \quad V_{\text {ref }}=\pi D \frac{n_{\mathrm{p}}}{60} \sqrt{1-\frac{n_{\mathrm{t}}}{n_{\mathrm{p}}}} \\
P_{\text {norm }}=\frac{P-P_{\text {hub }}}{P_{\text {ref }}}, \quad P_{\text {ref }}=\frac{1}{2} \rho V_{\text {ref }}^{2}
\end{gathered}
$$

where $D$ is the diameter of the torque converter, which is $245 \mathrm{~mm}$, and $P_{\text {hub }}$ is the static pressure measured at the stator hub.

The absolute stagnation pressure is defined as:

$$
\left(P_{O}\right)_{\mathrm{a}}=P+\frac{1}{2} \rho V_{O}^{2}
$$

where $\left(P_{o}\right)_{\mathrm{a}}$ is the absolute stagnation pressure, $P$ is the static pressure, and $V_{o}$ is the absolute total velocity.

In a rotating system, the rotary stagnation pressure is defined as:

$$
\begin{aligned}
\left(P_{o}\right)_{\mathrm{r}} & =P+\frac{1}{2} \rho W_{o}^{2} \\
P^{*} & =\left(P_{o}\right)_{r}-\frac{1}{2} \rho U^{2}
\end{aligned}
$$

where $U$ is the local blade speed, $P$ is the static pressure, and $W_{o}$ is the relative total velocity.

For incompressible, inviscid flow, the value of $P^{*}$ is constant along a streamline. For the viscous flow, the change of this parameter between two points on the same streamline represents the viscous loss.

\section{Unsteady Flow Field Data Processing}

The unsteady flow field data processing procedure used here is as follows:

(1) Calculate the instantaneous pressure of the five holes $\left(P_{1}, P_{2}, P_{3}, P_{4}, P_{5}\right)$ from the instantaneous voltages using the pressure transducer calibration curves.

(2) Calculate the instantaneous flow parameters $\left(\left(P_{o}\right)_{\mathrm{r}}, P,\left(W_{o}\right)_{\mathrm{t}}, W_{z}, \beta\right)$ from the pressure using the five-hole probe calibration.

(3) Decompose the instantaneous flow parameters into time mean average component, periodic component, and unsolved component using the phase lock averaging technique.

(4) Calculate the RMS (Root Mean Square) value and the associated unsteadiness quantity.

For the flow parameters in the turbine rotor passage, the instantaneous value $G_{i}$, is decomposed as follows:

$$
G_{i}=\overline{\bar{G}}+\tilde{G}+G^{\prime}
$$

where $\overline{\bar{G}}$ is the time-averaged value, $\tilde{G}$ is the periodic value, and $G^{\prime}$ is the unresolved flow component. The periodic unsteady component results from the relative motion of the rotor with respect to the stator/rotor, while the unresolved component is any flow field fluctuation that is not correlated with the rotor speed, such as turbulence and vortex shedding.

Since the data acquisition system is clocked by the shaft encoder, every instantaneous pressure measurement was recorded during each revolution. After all the instantaneous pressure measurements were acquired for the particular survey point, the instantaneous flow parameters were calculated from the pressure. Using the phase-lock averaging technique, the flow parameters were then ensemble averaged at each survey point of one revolution, according to:

$$
\bar{G}=\frac{1}{n} \sum_{i=1}^{n} G_{i}
$$


where $\bar{G}$ is the ensemble-averaged pressure, and $n$ is the total number of measurement in that particular survey point. The unresolved component for each flow parameter can then be calculated as

$$
G^{\prime}=G_{i}-\bar{G}
$$

The corresponding ensemble root mean square of the random fluctuation is calculated as

$$
\sqrt{G^{\prime 2}}=\sqrt{\frac{\left\{\sum_{i=1}^{n}\left(G_{i}-\bar{G}\right)^{2}\right\}}{(n-1)}}
$$

The level of unresolved unsteadiness in flow parameters is determined by this RMS value normalized by the corresponding property.

The time-averaged component $\overline{\bar{G}}$ is obtained by averaging all the ensemble-averaged flow parameters in each survey point as follows:

$$
\overline{\bar{G}}=\frac{1}{\mathrm{NR}} \sum_{j=1}^{\mathrm{NR}} \bar{G}_{j}
$$

where NR is the number of points per revolution. The time-averaged value is the time average of all measurements at a fixed point in space. The periodic component is then obtained from

$$
\tilde{G}=\bar{G}-\overline{\bar{G}}
$$

The RMS (Root Mean Square) of the periodic fluctuation can be calculated from the periodic component of flow parameters as follows:

$$
\sqrt{\tilde{G}^{2}}=\sqrt{\frac{\sum_{i=1}^{n} \tilde{G}^{2}}{(n-1)}}
$$

The periodic unsteadiness is calculated from the RMS (Root Mean Square) value of the periodic fluctuation normalized by the appropriate property. For the pressure and velocity quantity, the normalizing quantities are the total pressure drop through the turbine rotor passage and the mass averaged through flow velocity respectively. For the flow angle property, the normalizing quantity is the flow turning angle through the turbine rotor.

\section{RESULT AND DISCUSSIONS}

The frequency spectrums at speed ratio 0.6 at three typical measurement planes: turbine $1 / 4$ chord, mid-chord and 4/4 chord are shown in Figure 3. Only the spectrums of the pressure at the center

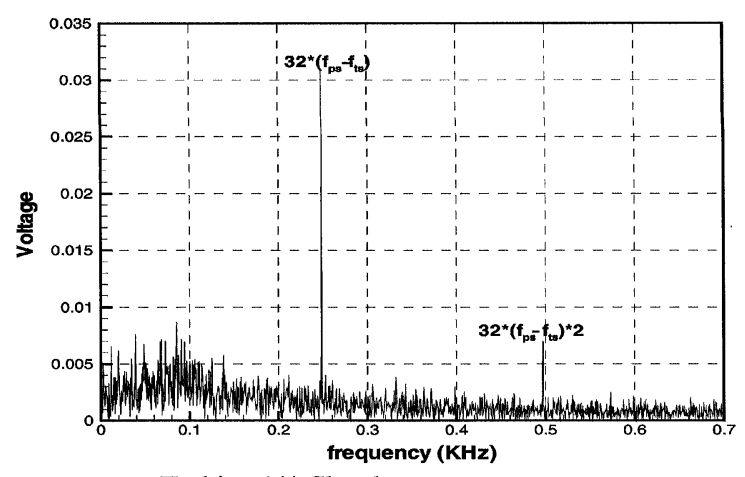

a. Turbine $1 / 4$ Chord

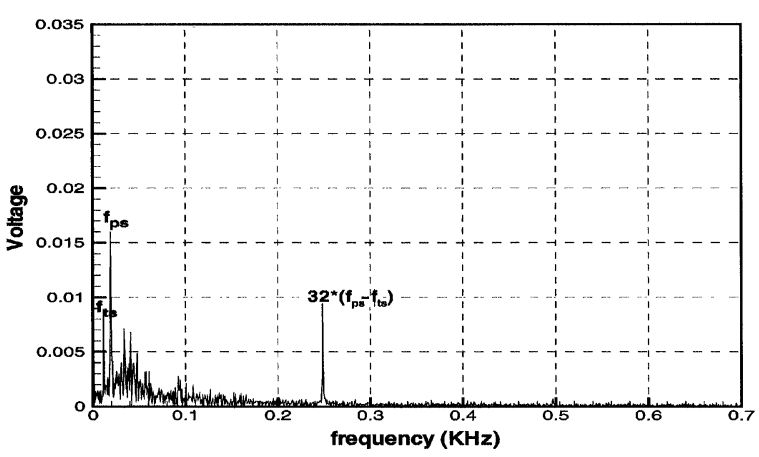

b. Turbine Mid-Chord

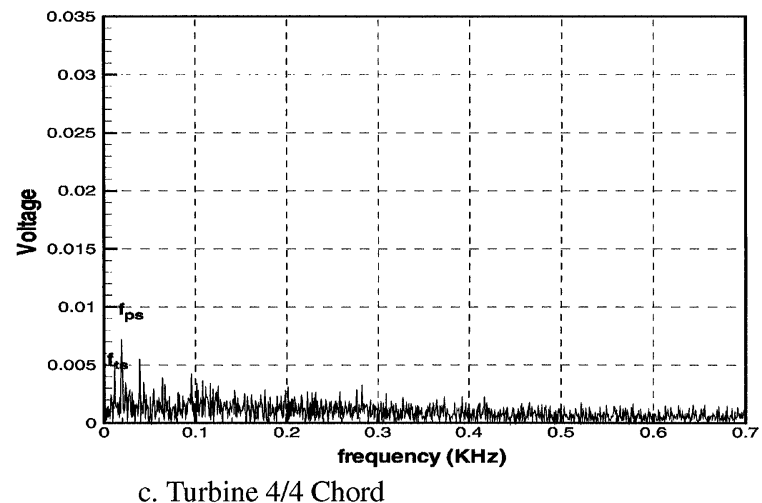

FIGURE 3 Frequency spectrums of rotating five-hole probe at center of turbine passage $(S R=0.6)$ 
hole (No. 1) are shown. The pump and the turbine shaft frequencies dominate the turbine passage at mid-chord and $4 / 4$ chord. This is mainly caused by the varying gravitational forces sensed by the probe during one revolution. The analysis of the data indicates that this is not due to vibration. The other dominant frequency is the pump blade passing frequency $\left(32^{*}(\mathrm{fps}-\mathrm{fts})\right)$ percieved in the turbine rotating frame. This represents the upstream effect of pump. The effect of the upstream pump diminishes very rapidly as the flow progresses from the turbine $1 / 4$ chord to the turbine trailing edge location. The absence of the stator passing frequency indicates that the influence of the downstream stator is negligible.

\section{Static Pressure $(P)$ Field}

The static pressure contours at the design condition $(S R=0.6)$ are shown in Figure 4. At the turbine $1 / 4$ chord, the static pressure has the highest value near the shell region, and the pressure gradient is mainly in the radial direction. This indicates that the blade loading is small at this location, which is consistent with blade static pressure measurements carried out by By and Lakshminarayana (1995). While at the turbine mid-chord, both the radial and the tangential pressure gradients dominate. At the turbine $4 / 4$ chord, again the pressure gradient is in the radial direction, but the gradient is much smaller than the gradient at the turbine $1 / 4$ chord.

In centrifugal turbomachines, both the effects of rotation and curvature are important. The curvature terms include both the radius of curvature due to the sloping path or curvature of meridional streamline and curvature due to swirling and rotating flows. The equilibrium for an invisid flow in the meridional plane is described by the following equation:

$$
\frac{1}{\rho} \frac{\partial P}{\partial z}=\frac{V_{m}^{2}}{R_{z}}-\frac{V_{u}^{2}}{r} \cos \varepsilon
$$

where $P$ is the static pressure, $V_{m}$ is the meridional velocity, $R_{z}$ is the radius of meridional curvature, $r$

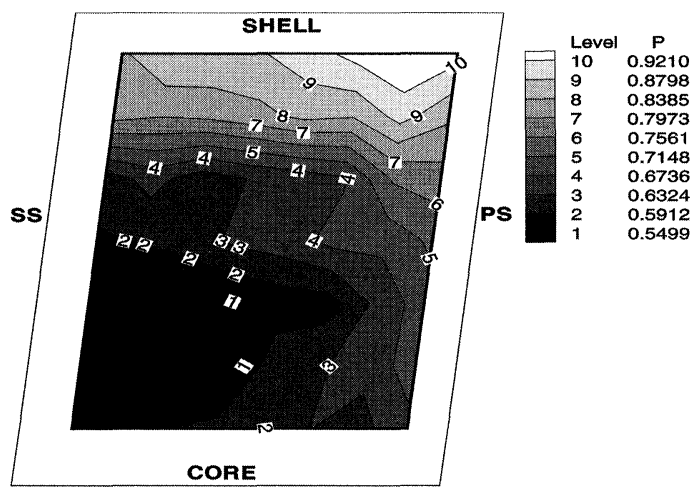

a. Turbine $1 / 4$ Chord

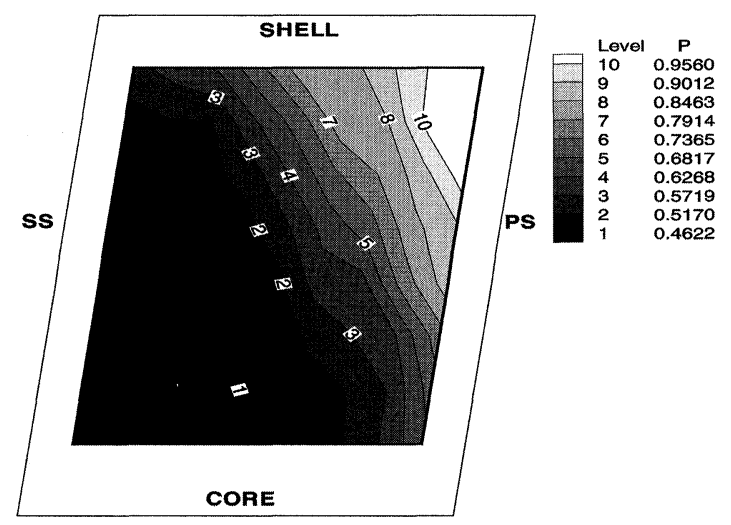

b. Turbine Mid-Chord

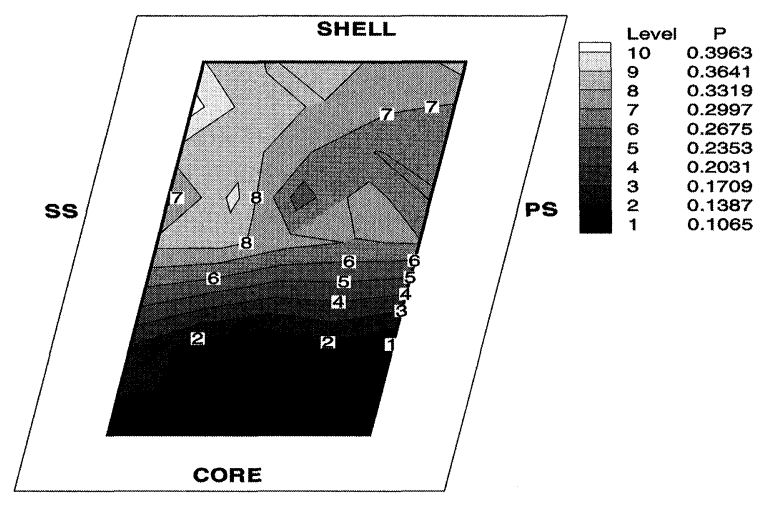

c. Turbine $4 / 4$ Chord

FIGURE 4 Contour plots of normalized static pressure $P$ $(S R=0.6$, Eq. 2).

is the radius of swirling flow, and $\varepsilon$ is the angle between turbine axis and the line perpendicular to the meridional line. 
From this equilibrium equation, $\varepsilon$ is greater than $90^{\circ}$ at the turbine $1 / 4$ chord. This indicates that the effects of centrifugal force due to swirling flow and the meridional curvature results in the observed radial static pressure gradient. At the turbine mid-chord, the contribution of the centrifugal force due to the swirling flow to the radial pressure gradient is insignificant, because the difference in radius at the core and shell is very small. Thus, the radial pressure gradient at the turbine mid-chord is not as significant as that at the turbine $1 / 4$ chord. Finally, at the turbine $4 / 4$ chord, the effects of centrifugal force due to swirling and meridional curvature cancel each other, and the radial pressure gradient is even smaller than that at the turbine mid-chord.

\section{Absolute Stagnation Pressure $\left(\boldsymbol{P}_{\boldsymbol{o}}\right)_{\mathbf{a}}$ Field}

The contour plots of the absolute stagnation pressure are shown in Figure 5. At this speed ratio, the absolute stagnation pressure contour at the turbine $1 / 4$ chord location (Fig. 5a) show that the pressure has the highest value near the shell, and the pressure gradient is mainly in the radial direction, from the shell to the core. On the other hand, at the turbine mid-chord, both radial and tangential gradient dominate. At the turbine $4 / 4$ chord, the radial pressure gradient dominates.

Furthermore, from these three figures (Figs. $5 \mathrm{a}-\mathrm{c})$, it can also be seen that the absolute stagnation pressure drop is evenly distributed from the turbine $1 / 4$ chord to the turbine $4 / 4$ chord. This is different from the static pressure drop, because the flow turning plays a very important role in absolute stagnation pressure drop.

The mass-averaged absolute stagnation pressure drop from the turbine inlet to the turbine exit is shown in Figure 6a. It is clear that the absolute stagnation pressure drop is almost evenly distributed along the turbine flow path. This even distribution should be expected, as this is the design condition. The incidence angle is relative small and the pump/turbine matches relatively well

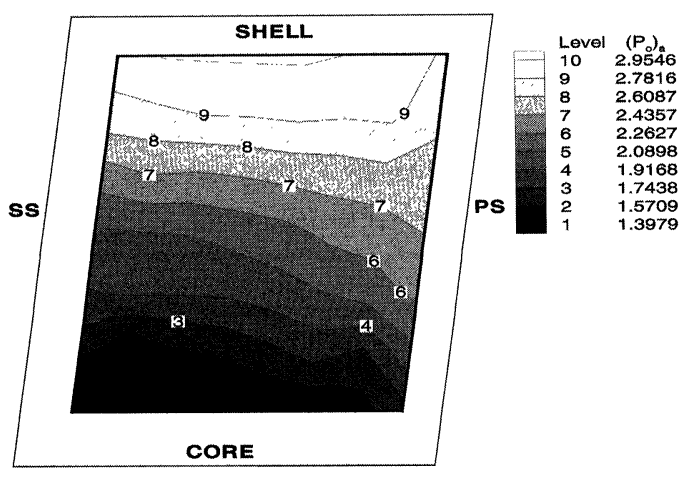

a. Turbine $1 / 4$ Chord

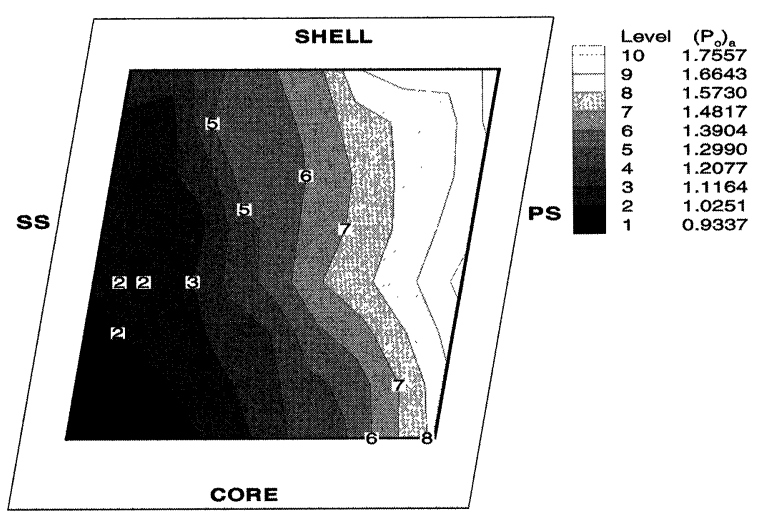

b. Turbine Mid-Chord

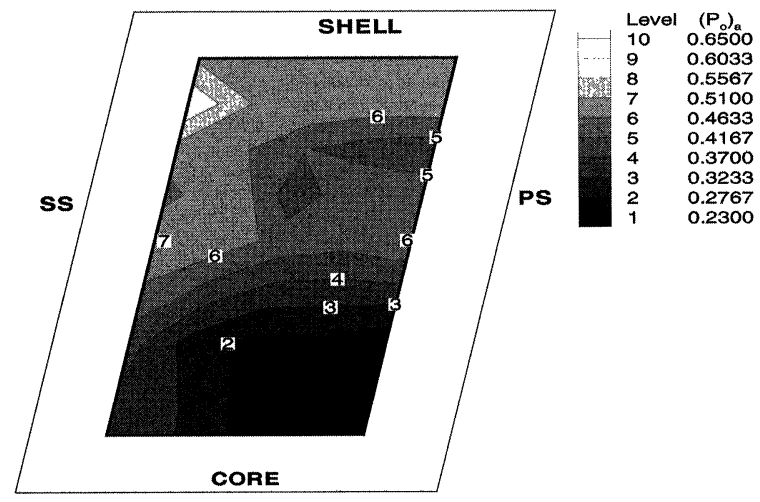

c. Turbine $4 / 4$ Chord

FIGURE 5 Contour plots of normalized absolute stagnation pressure $\left(P_{o}\right)_{\mathbf{a}}(S R=0.6$, Eq. 3$)$.

at this operating condition. There is a pressure loss from the turbine trailing edge to the exit measurement location. 

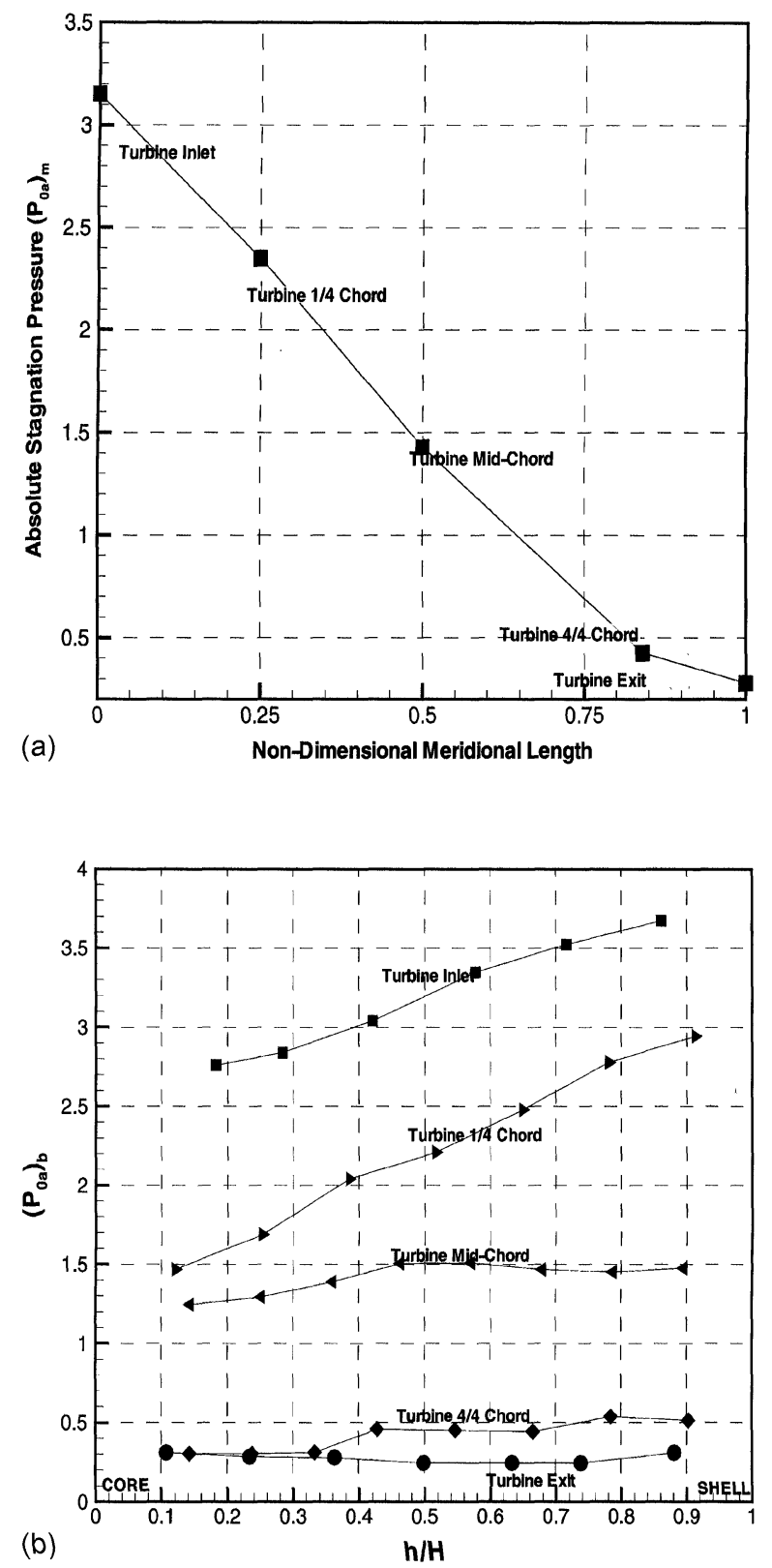

FIGURE 6 Absolute stagnation pressure distribution $(S R=0.6)$. (a) Mass averaged absolute stagnation pressure $\left(P_{o \mathrm{a}}\right)_{\mathrm{m}}$ drop $(S R=0.6)$; (b) Radial distribution of blade-toblade averaged absolute stagnation pressure $\left(P_{o \mathrm{a}}\right)_{\mathrm{b}}(S R=0.6)$.

The radial distribution of blade-to-blade mass averaged absolute stagnation pressure from the turbine inlet to the turbine exit $(S R=0.6)$ is shown in Figure $6 b$. It is clear that the turbine receives a much higher total pressure at the shell than at the core. This is caused by the nearly separated flow near the core at the pump exit.

In addition, there is approximately $50 \%$ greater pressure drop near the shell as compared to the core. Also there is a greater drop associated with the first half of the turbine passage duct, particularly near the shell. This can be attributed to the inlet flow field, with high pressure and velocity fluid near the shell. It can also be seen that the total pressure in the second half of the turbine is much more uniform from core to shell than is the first half. The possible cause is the intense mixing caused by the secondary flow, which tends to provide more uniform pressure distribution at the exit.

Furthermore, there is some pressure drop from the turbine $4 / 4$ chord to the turbine exit, this represents the pressure loss due to the trailing-edge wake decay, dissipation of the secondary kinetic energy as well as turbulence kinetic energy. All these factors could collectively result in pressure loss downstream of the turbine trailing edge. The pressure loss is high near the shell and low near the core region, this is due to high velocity and high pressure near the shell at the turbine trailing edge location and this gives high mixing losses.

\section{Relative Total Velocity $\left(W_{o}\right)_{t}$ Field}

The contour plots of the relative total velocity are shown in Figure 7 . At the turbine $1 / 4$ chord, the relative total velocity is high near the shell and low near the core/suction corner. The flow is nearly uniform in the blade-to-blade direction. A low velocity region is observed near the core. This is mainly caused by the non-uniform flow at the exit of the pump, where low velocity is observed near the core (Dong, 1998). At the turbine mid-chord, high velocity fluid is located near the pressure side/ shell corner, and low velocity fluid is concentrated near the suction side. At this location, the velocity is very low and could be separated. From this point of view, the low velocity region near the core/suction corner at the turbine $1 / 4$ chord may also be a separated area. The secondary flow transports this flow from the core region at the 


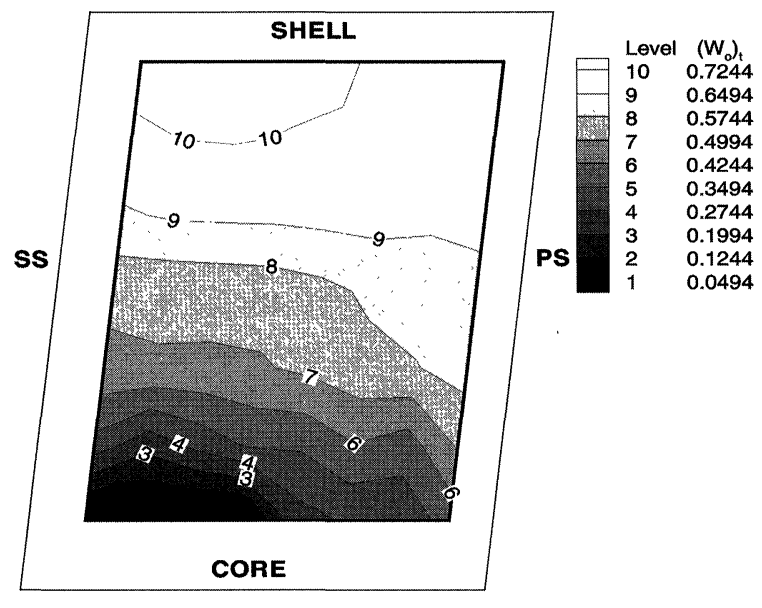

a. Turbine $1 / 4$ Chord

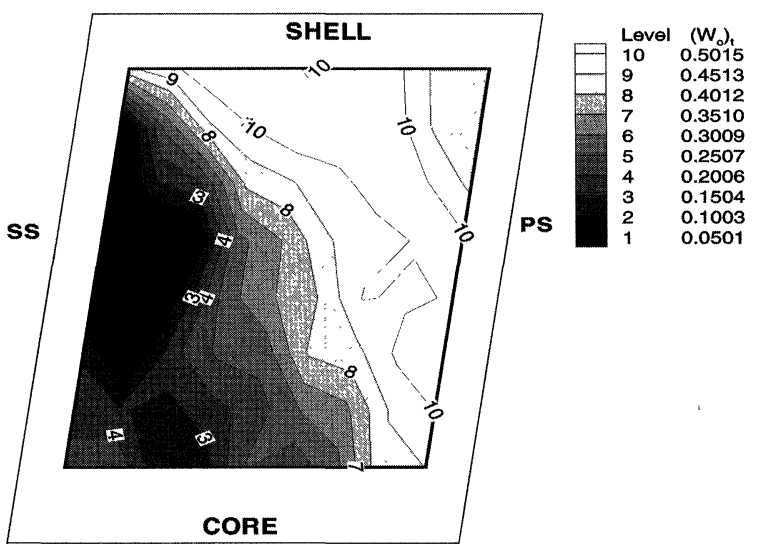

b. Turbine Mid-Chord

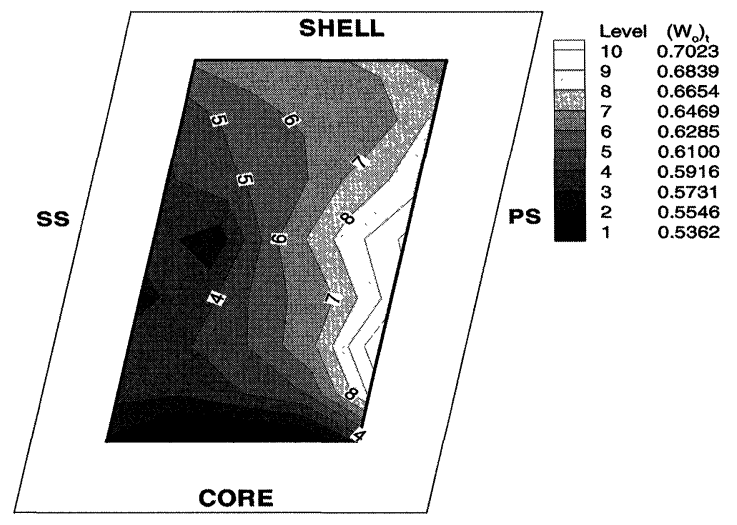

c. Turbine $4 / 4$ Chord

FIGURE 7 Contour plots of normalized relative Total velocity $\left(W_{0}\right)_{\mathrm{t}}(S R=0.06$, Eq. 1$)$. turbine $1 / 4$ chord to the suction side at the turbine mid-chord. At the turbine $4 / 4$ chord, the velocity is relatively uniform, the low velocity region observed earlier has been eliminated.

It is clear by comparing the data in Figure 4, 5 and 7 that the static pressure drop and relative velocity change from the turbine $1 / 4$ chord to the mid-chord is not significant, while the stagnation pressure drop is substantial (approximately 50\% drop in normalized total pressure). This shows the major influence of radius change and the rotation effect (Coriolis and centrifugal forces). The flow turning effect is augmented substantially by these rotation effects.

The radial distributions of blade-to-blade relative total velocity from the pump exit to the turbine exit are shown in Figure 8. It is clear that the change in relative total velocity from the pump exit to the turbine exit is relatively small. The drop in absolute stagnation pressure is caused predominantly by the centrifugal and the Coriolis forces and not by the acceleration of the relative flow. The flow is highly non-uniform in the radial direction at the turbine $1 / 4$ chord and turbine mid-chord, which becomes nearly uniform at the turbine $4 / 4$ chord and the turbine exit. This may be attributed to possible flow separation near the core/suction corner at the turbine $1 / 4$ chord and the turbine mid-chord.

The most noticeable feature is that the turbine inlet velocity is significantly higher than those at the other four planes. It is important to recall that the turbine inlet measurements were taken in the gap between the pump and turbine and not at the leading edge of the turbine. The higher relative total velocity can be explained by considering velocity triangles. The total velocity still contains a large tangential component created by the pump, which has a higher rotational speed. Thus, there is high angular momentum at the turbine inlet. At the turbine exit, the turbine also imparts a high relative tangential velocity. Thus, the relative total velocity would be high at the exit as well. However, the turbine rotates much more slowly than the pump, and the velocities are not as high as the inlet velocities. 


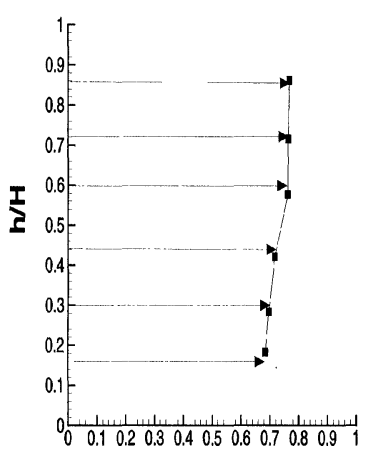

$\left(W_{0}\right)_{b}$

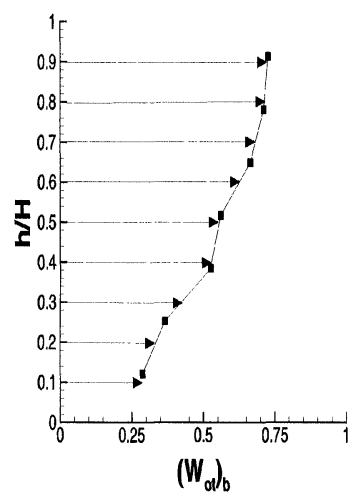

b. Turbine $1 / 4$ Chord

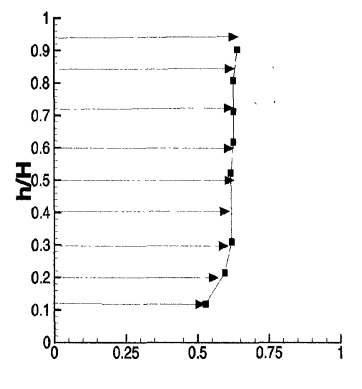

$\left(W_{0}\right)_{b}$

d. Turbine $4 / 4$ Chord

\section{c. Turbine Mid-Chord}

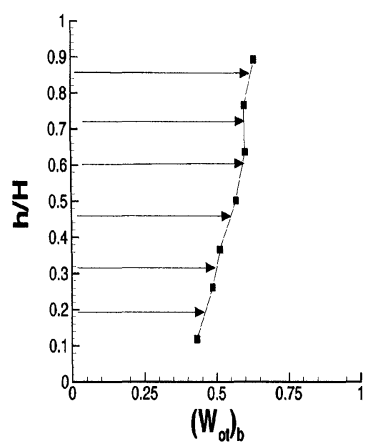

e. Turbine Exit

FIGURE 8 Radial distribution of blade-to-blade averaged relative total velocity $\left(W_{o t}\right)_{\mathrm{b}}(\mathrm{SR}=0.6$, Eq. 1$)$.

\section{Through Flow Velocity $\left(W_{z}\right)$ Field}

The contour plots of through flow velocity are shown in Figure 9. The through flow velocity is the velocity component perpendicular to the measurement plane.

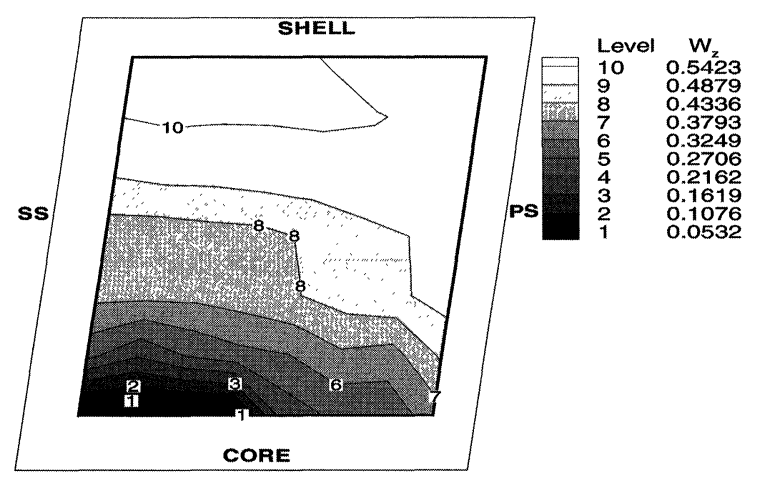

a. Turbine $1 / 4$ Chord

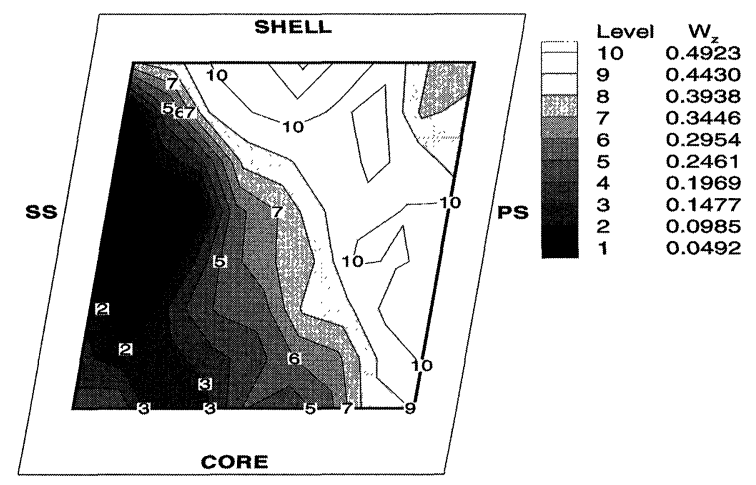

b. Turbine Mid-Chord

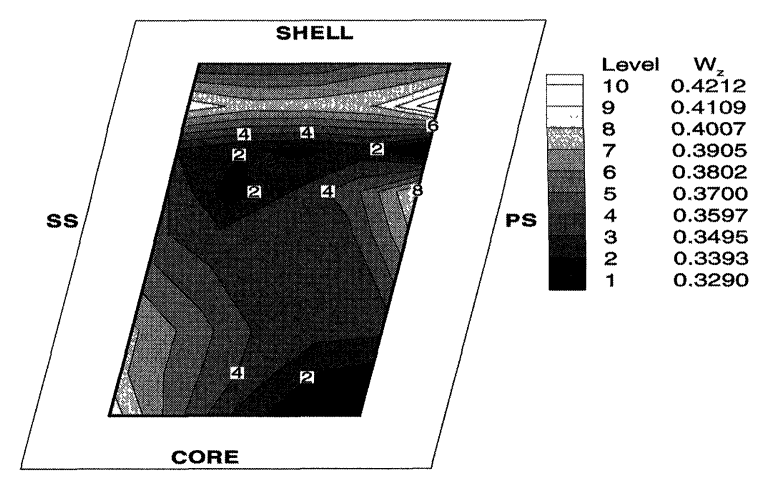

c. Turbine $4 / 4$ Chord

FIGURE 9 Contour plots of though flow velocity $\left(W_{z}\right)$ $(S R=0.6$, Eq. 1).

The overall trend in the through flow velocity distribution is very similar to that of the total velocity field (Fig. 7). A "jet-wake" flow structure is observed at the turbine $1 / 4$ chord and the turbine mid-chord. At the turbine $1 / 4$ chord, mass flow is concentrated near the shell region, and a 
possible flow separation area is observed near the core/suction side corner. At the turbine mid-chord, a different "jet-wake" flow pattern exists. High velocity fluid is observed near the pressure side/ shell corner, and low velocity fluid (wake) region is located near the middle of suction side. However, at the turbine $4 / 4$ chord (turbine trailing edge location), the through flow is very uniform and no flow separation area exists.

One reason for the low velocity region at the turbine $1 / 4$ chord (Figs. 7 and 9) is the pump exit flow and the high incidence angle at the turbine leading edge. Due to the "jet-wake" flow structure inside the pump passage, the velocity is high near the shell and very low near the core region at the pump exit. In addition, the incidence angle at the turbine leading edge is about $15^{\circ}$. This probably causes flow separation at the core/suction side corner at the turbine $1 / 4$ chord. As the flow progresses from the turbine $1 / 4$ chord to the turbine mid-chord, the meridional curvature and camber curvature effect begins to dominate. The secondary flow tends to redistribute the flow field. This may cause the low velocity region to move from the core to the suction side as the flow progresses. As the flow continues from the turbine mid-chord to the turbine trailing edge, the meridional curvature effect continues to influence the flow field. The flow near the core region accelerates due to the convex curvature, and the flow near the shell region decelerates. As a result of this, flow reattaches before the turbine trailing edge near the core. This is also augmented by the secondary flow.

\section{Relative Flow Yaw Angle $\left(\beta_{t}\right)$ Field}

The contour plots of the yaw angle measured by the rotating five-hole probe are shown in Figure 10. At the turbine $1 / 4$ chord, the blade angle is $48.12^{\circ}$, and at the turbine mid-chord it is $0^{\circ}$. The large overturning at the turbine $1 / 4$ chord can be attributed to large positive incidence and mismatch between the pump exit flow and turbine inlet flow. The core flow region has very low

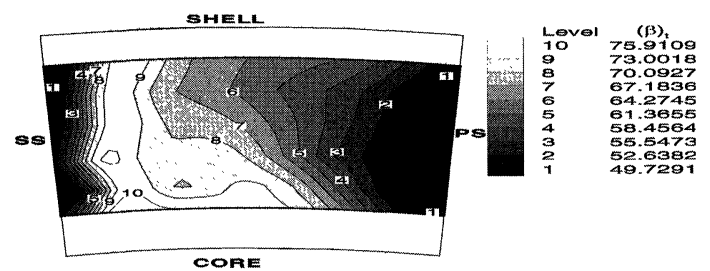

a. Turbine Inlet

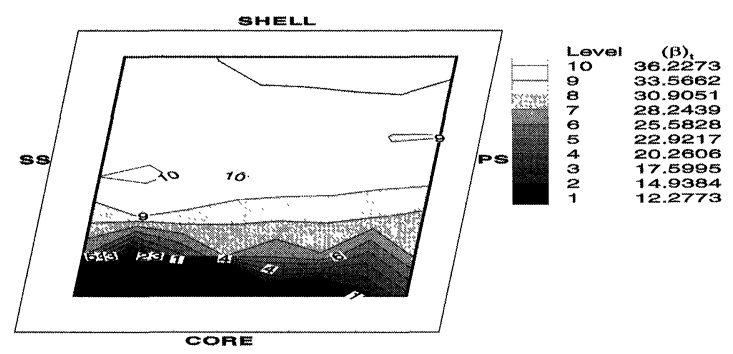

b. Turbine $1 / 4$ Chord

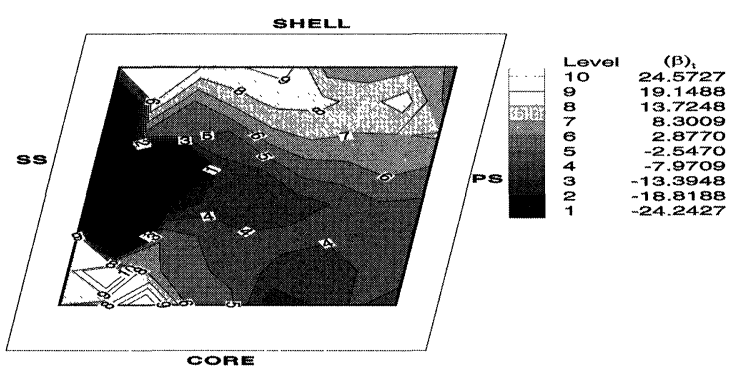

c. Turbine Mid-Chord

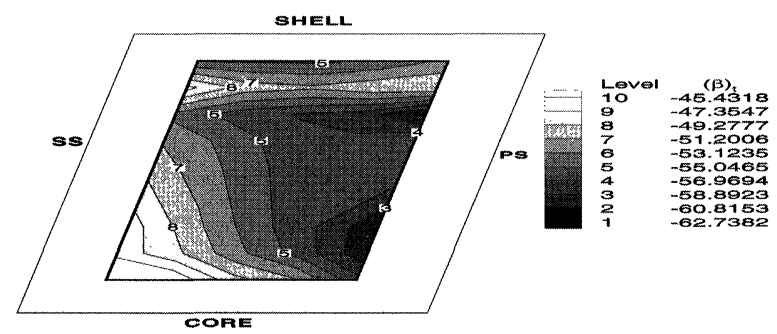

d. Turbine $4 / 4$ Chord

FIGURE 10 Contour plots of relative flow Yaw Angle $\left(\beta_{\mathrm{t}}\right)$ $(S R=0.6)$.

through flow and total relative velocities and operates at substantial off-design condition with flow angle ranging from $12^{\circ}$ to $20^{\circ}$. This off-design condition is located near the suction side at the turbine mid-chord. Most of the core flow has 
relative flow angle from $-2^{\circ}$ to $8^{\circ}$. The flow is underturned in most regions, even though the pressure drop is substantial (Fig. 5); confirming the earlier conclusion regarding the dominant effect of centrifugal and Coriolis forces.

The blade angle at the turbine $4 / 4$ chord is $-58.47^{\circ}$, and the relative flow yaw angle contour plot at this location shows that the flow is well aligned with the turbine blade. The maximum deviation angle is about $11^{\circ}$ near the core/suction corner and the middle of suction side. The presence of large deviation angle at these two areas can be attributed to the residual effect of possible flow separation, which plays a very significant role at the turbine $1 / 4$ chord and turbine mid-chord.

\section{Rotary Stagnation Pressure $\left(P^{*}\right)$ Field}

The contour plots of rotary stagnation pressure are shown in Figure 11. The difference in rotary stagnation pressure along flow path represents losses. The contour plots shown in Figure 11 indicate that these values are low near the core and high near the shell. This is partially attributed to pump exit flow and not the losses within the turbine passage. On the other hand, the difference in these values between 1/4 chord and mid-chord represents flow losses inside the turbine passage. The flow losses are highest near the blade suction side and quite significant near the shell region.

The rotary stagnation pressure decreases near the shell region and increases near the core region from the turbine mid-chord to the turbine $4 / 4$ chord. The increase in rotary stagnation pressure near the core region is due to the flow reattachment before the turbine trailing edge. Because of the flow reattachment, the high mixing losses near the separation region are substantially reduced.

Overall, it can be concluded from the rotary stagnation pressure contour plots that there are higher losses at the first half of the turbine passage than the second half, and possible flow separation is the major sources of loss.

The radial distributions of blade-to-blade averaged relative total pressure $\left(P_{o r}\right)_{\mathrm{b}}$ from the pump

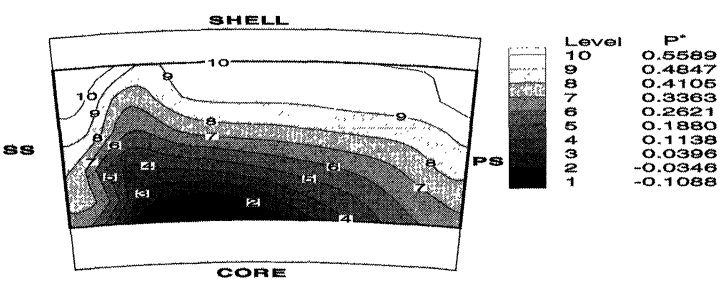

a. Turbine Inlet

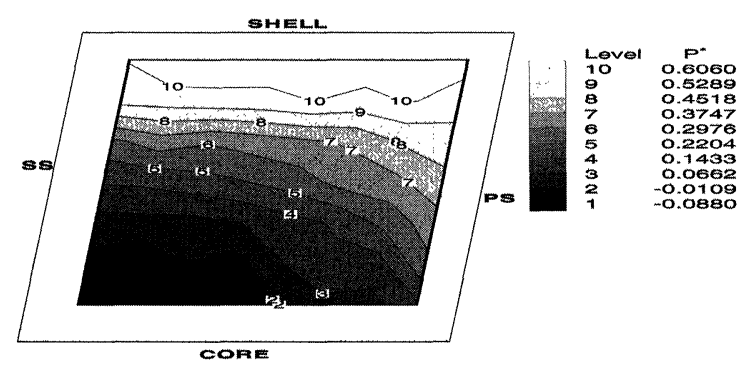

b. Turbine $1 / 4$ Chord

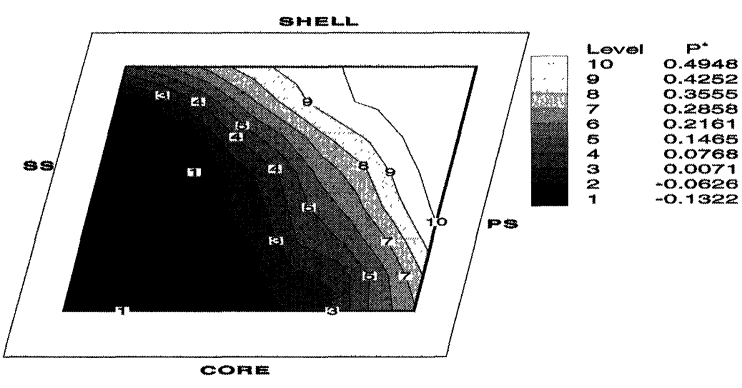

c. Turbine Mid-Chord

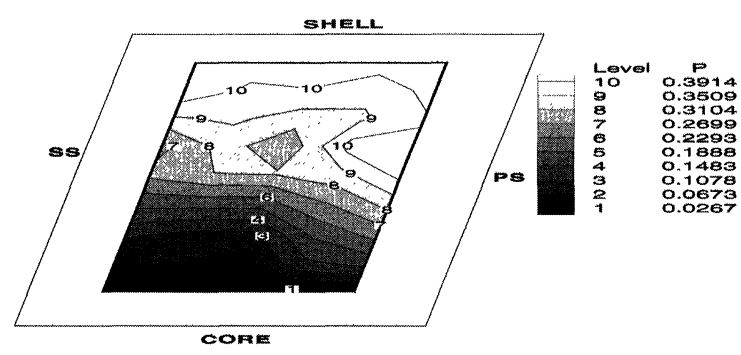

d. Turbine $4 / 4$ Chord

FIGURE 11 Contour plots of rotary stagnation pressure $\left(P^{*}\right)$ $(S R=0.6$, Eq. 4).

exit to the turbine exit are shown in Figure 12. It is clear that the relative total pressure decreases as the flow progresses from inlet to exit. Examining the radial distribution of relative total velocity 


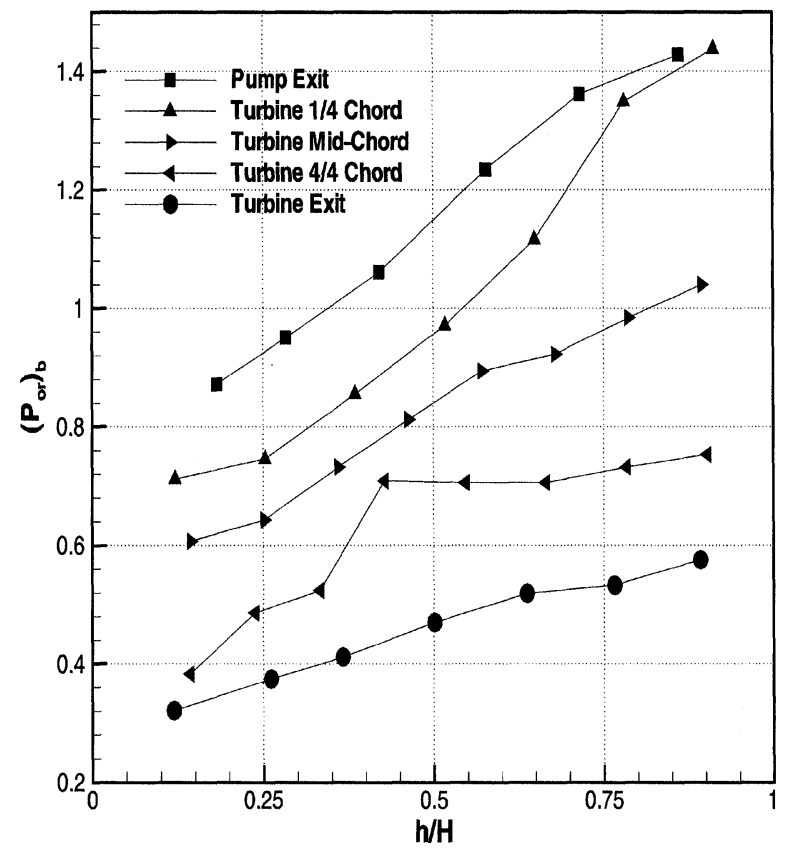

FIGURE 12 Radial distribution of blade-to-blade averaged relative total pressure $\left(P_{o r}\right)_{\mathrm{b}}(S R=0.6)$.

(Fig. 8), it is concluded that the drop in relative stagnation pressure is mainly caused by the drop in static pressure, not by the change in the relative total velocity.

In addition, the relative stagnation pressure is low near the core region at all measurement planes. This feature may be attributed to several factors. First, this low stagnation pressure may be caused by the "jet-wake" flow structure at the pump exit, which causes low pressure rise near the core region inside the pump passage. Second, the low stagnation pressure near the core is attributed to the low mass flow near the core region, which is typical in mixed flow turbomachines. Finally, there exists flow separation near the core region. This causes higher losses, resulting in low stagnation pressure near the core region.

\section{Blade-to-blade Averaged Rotary Stagnation Pressure $\left(\boldsymbol{P}^{*}\right)_{\mathrm{b}}$}

The radial distribution of blade-to-blade averaged rotary stagnation pressure $\left(P^{*}\right)_{\mathrm{b}}$ for speed ratio 0.6 is shown in Figure 13. This quantity represents the

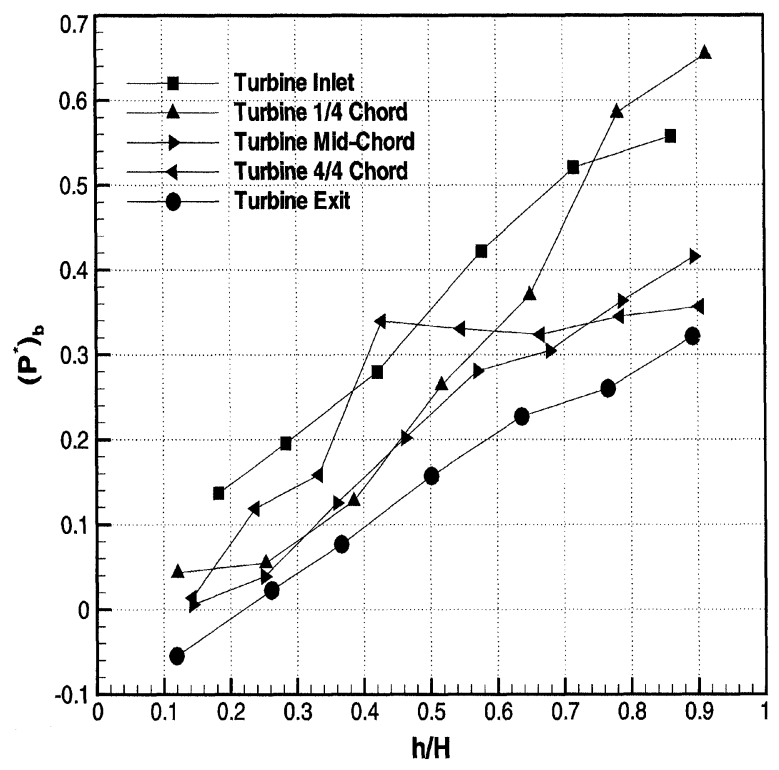

FIGURE 13 Blade-to-blade averaged rotary stagnation pressure $\left(P^{*}\right)_{\mathrm{b}}(S R=0.6)$.

difference between local stagnation pressure and the stagnation pressure calculated from the inviscid Euler equation. This quantity should remain nearly constant in an inviscid flow, and the difference in this quantity between any two stations represents the hydrodynamic loss. Since a decrease in this quantity represents a pressure loss, it is clear that there are greater losses occurring in the first half of the turbine passage. This is particularly true in the end-wall regions. The flow separation is most likely the source of these losses. Though the drop in rotary stagnation pressure is much smaller in the second half of the passage duct, it is still greatest in the mid-span and shell regions. However, because the flow reattaches before the turbine trailing edge, and because the second half of the passage duct does not experience the flow disturbances associated with the inlet, the losses are smaller in the second half of the turbine.

\section{Blade-to-blade Periodic Unsteadiness in Relative Pressure, Velocity, and Flow Angle}

The radial distributions of blade-to-blade averaged periodic unsteadiness at the turbine $1 / 4$ 
chord, $2 / 4$ chord, and turbine $4 / 4$ chord are shown in Figures 14, 15 and 16 respectively. The periodic unsteadiness in $P_{o}, P, W_{o}$, near the core region $(h /$ $H=0-30 \%$ ) at the turbine $1 / 4$ chord is quite high $(7-10 \%)$ and low near the shell region (about $2.5 \%)$. This can be attributed to the flow separation near the core/suction side corner at the turbine $1 / 4$ chord. At this area, the fluctuations in all the flow parameters (pressure, velocity, and flow angles) are quite high. The unsteady level in the free stream region $(h / H=30-100 \%)$ is relatively low and uniform. There exists a region showing high fluctuations at the turbine $2 / 4$ chord, which is located near the mid-span $(h / H=55-$ $80 \%$ ). The unsteady level is about $2-5$ times of those observed in the free stream region. This can also be explained by the possible flow separation and the associated high fluctuations near the suction side at the turbine $2 / 4$ chord. On the other hand, the radial distribution of the periodic unsteadiness at the turbine $4 / 4$ chord is relatively uniform from the core $(2.5 \%)$ to the shell (about $1.5 \%)$

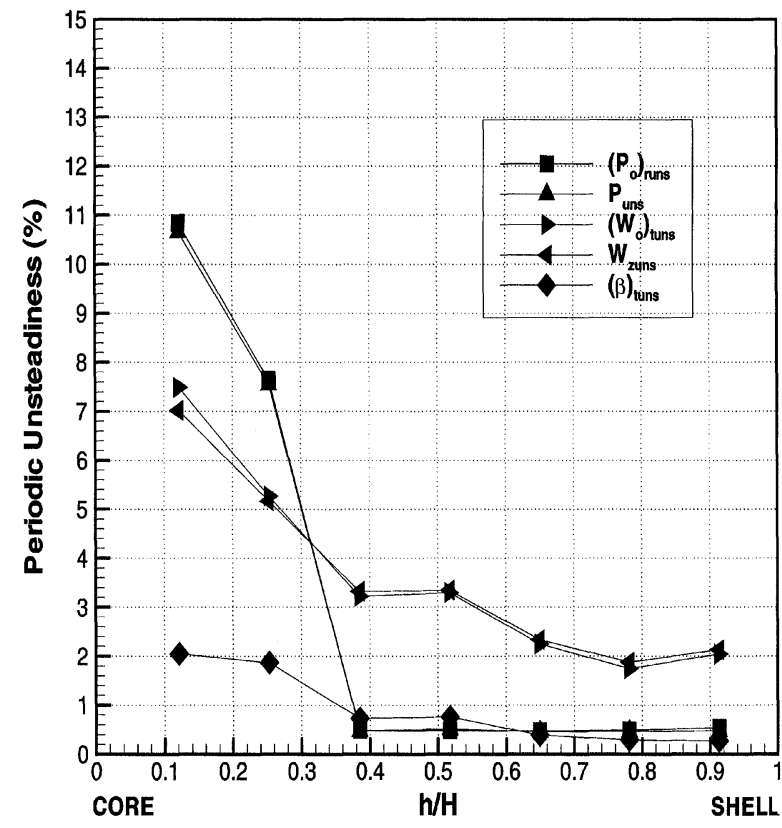

FIGURE 14 Radial distribution of blade-to-blade averaged periodic unsteadiness in flow field $(S R=0.6$, Turbine $1 / 4$ Chord).

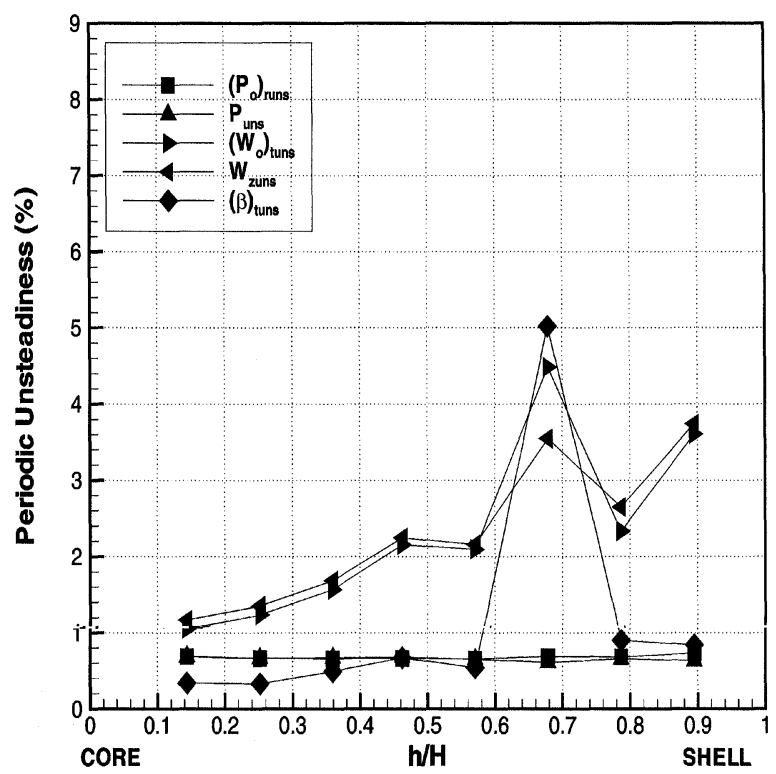

FIGURE 15 Radial distribution of blade-to-blade averaged periodic unsteadiness in flow field $(S R=0.6$, Turbine $2 / 4$ Chord).

The higher unsteadiness at the turbine $1 / 4$ chord and turbine $2 / 4$ chord is due to the following reasons. First, the measurement planes at the turbine $1 / 4$ chord and turbine $2 / 4$ chord are much closer to the pump trailing edge than the turbine

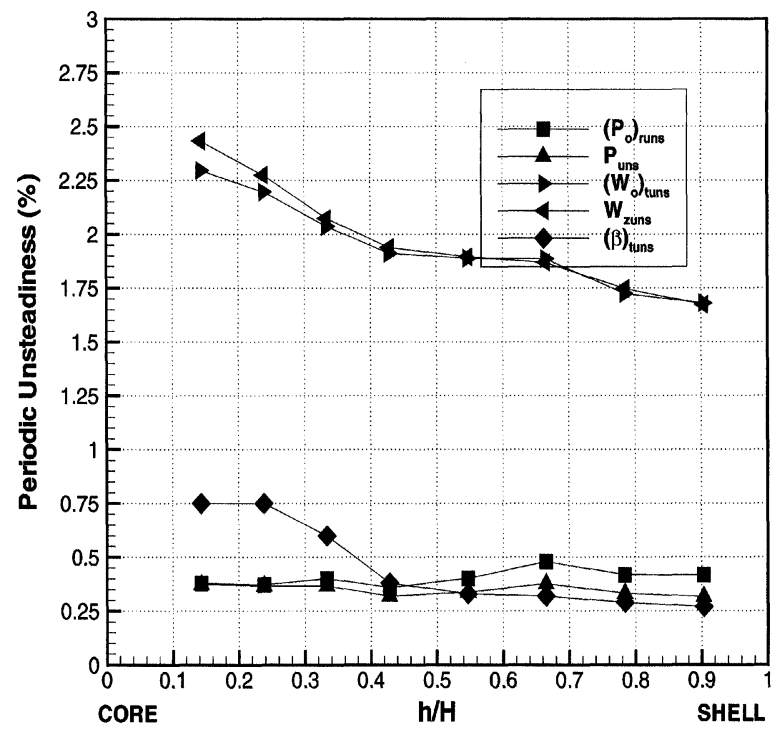

FIGURE 16 Radial distribution of blade-to-blade averaged periodic unsteadiness in flow field $(S R=0.6$, Turbine $4 / 4$ Chord). 
$4 / 4$ chord. Hence, the pump blade wake has not decayed completely at these two planes, and has significant effect on the unsteady flow field. Secondly, there exist large pressure gradients as shown in Figure 4. The pressure gradient is from the shell to the core and from the pressure side to the suction side for the turbine $1 / 4$ chord and $2 / 4$ chord respectively. These pressure gradients drive the secondary flow from the high pressure region to the low pressure area, this results in the accumulation of low momentum fluid at the suction/core corner at the turbine $1 / 4$ chord and near the suction side at the turbine $2 / 4$ chord. At the turbine $4 / 4$ chord, however, the static pressure is very uniform. There is no clear pressure gradient and no low momentum fluid region. This can explain why the unsteadiness near the turbine trailing edge is low and uniform.

The basic feature of the periodic unsteadiness in relative pressure, velocity, and flow angle, is quite similar at these three measurement planes (Figs. 14, 15 and 16). That is, the high periodic unsteadiness region is limited to the core/suction corner at the turbine $1 / 4$ chord, and the suction side region at the turbine $2 / 4$ chord. On the other hand, the unsteady level is relatively low and uniform at the turbine $4 / 4$ chord. This agrees well with the through flow velocity distribution shown in Figure 9. The magnitude of the unsteady level in the free stream regions is only about $20-30 \%$ of that observed in the flow separation region. This indicates that the low pressure, low velocity region may be separated, and these may be the regions that contribute to the high losses and inefficiency.

\section{CONCLUSION}

From the data presented at the design condition $(S R=0.6)$, the following conclusions can be drawn:

(1) Upstream pump exit flow field has a dominant effect on the flow field in the entrance region of the turbine passage; however, these effects diminish very rapidly as the flow progresses from the turbine $1 / 4$ chord to the turbine trailing edge.

(2) The spectrum analysis reveals the presence of flow unsteadiness at the pump blade passing frequency near the turbine $1 / 4$ chord, with insignificant trace at the turbine mid-chord. The spectrum at the turbine exit shows neither the pump nor the downstream stator influence on the turbine exit flow field.

(3) The static pressure gradient is mainly in the radial direction at the turbine $1 / 4$ and $4 / 4$ chord. While at the turbine mid-chord, both the radial and the tangential pressure gradient dominant. This static pressure drop is caused by both the flow turning and the effects of rotation and curvature.

(4) The mass averaged stagnation pressure drop is almost evenly distributed along the turbine flow path. In addition, there is approximately $50 \%$ greater pressure drop near the shell as compared to the core. This can be attributed to the inlet flow field which has high pressure and velocity near the shell.

(5) Examining the static pressure, relative velocity, and absolute stagnation pressure field, it is concluded that the drop in absolute stagnation pressure is caused predominantly by the centrifugal and the Coriolis forces and not by the acceleration of the relative flow. The flow turning effect is augmented substantially by the rotation effects.

(6) The rotary stagnation pressure distribution indicates that there are a lot of losses occurring in the first half of the turbine passage. The flow separation and the mismatch between the pump exit and turbine inlet flow field are the most likely sources of these high losses.

(7) The main feature of the unsteady flow in the shell region is that the flow fluctuation is relatively low and uniform. The unsteady levels are much lower than that observed in the corner flow separation, or flow mixing areas.

(8) The core region is the most inefficient region due to possible flow separation. Pump/turbine 
mismatch is another source of inefficiency of turbine. The efficiency and fuel economy can be improved through a redesign of the core region and by improved matching of the pump exit flow and the turbine inlet flow field.

\section{Acknowledgements}

This project was sponsored by the Powertrain Division of the General Motors Corporation. The authors wish to express their gratitude to $\mathrm{Mr}$. Donald G. Maddock of GM for his assistance, comments, and encouragement. Assistance by Y. Dong is gratefully acknowledged.

\section{NOMENCLATURE}

$\begin{array}{ll}A & \text { area } \\ D & \text { diameter of torque converter } \\ f_{\mathrm{p}, \mathrm{s}} & \text { pump shaft frequency }\left(n_{\mathrm{p}} / 60\right) \\ f_{\mathrm{ts}} & \text { turbine shaft frequency }\left(n_{\mathrm{t}} / 60\right) \\ G & \text { flow parameter } \\ h & \text { probe radial height measured from core, } \\ & \text { mm } \\ H & \text { blade height, mm } \\ h / H & \text { relative radial position }(=0 \text { at core, }=1 \\ & \text { at shell) } \\ n_{\mathrm{p}} & \text { pump rotating speed } \\ n_{\mathrm{t}} & \text { turbine rotating speed } \\ P & \text { static pressure } \\ P_{\mathrm{hub}} & \text { static Pressure at hub } \\ P_{\text {norm }} & \text { normalized Pressure }(\text { Eq. }(2)) \\ P_{\text {ref }} & \text { reference pressure }(\text { Eq. }(2)) \\ \left(P_{o}\right)_{\mathrm{a}} & \text { absolute stagnation pressure }(\text { Eq. }(3)) \\ \left(P_{o \mathrm{a}}\right)_{\mathrm{b}} & \text { blade-to-blade averaged absolute stagna- } \\ & \text { tion pressure } \\ \left(P_{o \mathrm{a}}\right)_{m} & \text { mass averaged absolute stagnation } \\ & \text { pressure } \\ \left(P_{o}\right)_{\mathrm{r}} & \text { relative total pressure }(\text { Eq. }(4)) \\ P^{*} & \text { rotary stagnation pressure }(\text { Eq. }(4)) \\ \mathrm{P} . \mathrm{S} & \text { pressure side } \\ \mathrm{SR} & \text { speed ratio, } n_{\mathrm{t}} / n_{\mathrm{p}} \\ \mathrm{S} . \mathrm{S} & \text { suction side }\end{array}$

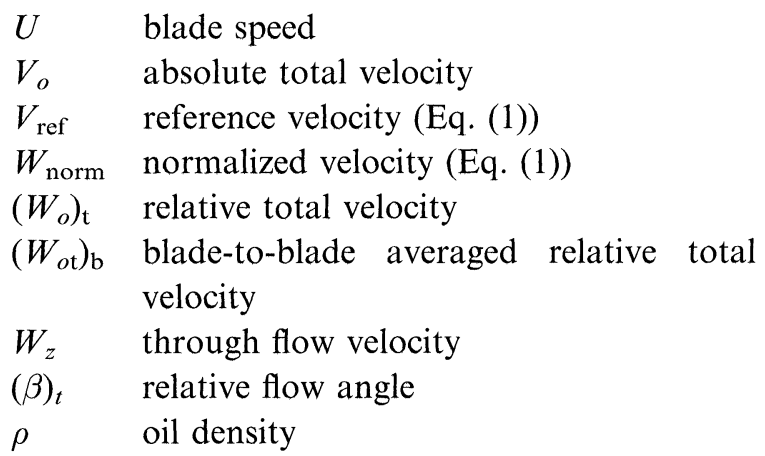

\section{Subscript}

b, $m$ blade-to-blade averaged, mass averaged uns periodic unsteadiness

\section{Superscript}

$\begin{array}{ll}- & \text { Ensemble Averaged } \\ = & \text { Time Averaged } \\ \sim & \text { Periodic } \\ , & \text { Unresolved }\end{array}$

\section{References}

Browarzik, V. (1994) "Experimental Investigation of Rotor/ Rotor Interaction in a Hydrodynamic Torque Converter Using Hot-Film Anemometry", ASME Paper No. 94-GT246, presented at the International Gas Turbine and Aeroengine Congress and Exposition, The Hague, Netherlands, June 13-16.

Brun, K. and Flack, R. D. (1997a) "Laser Velocimeter Measurement in the Turbine of an Automotive Torque Converter: Part I-Average Measurements", ASME Journal of Turbomachinery, 119, 646-654.

Brun, K. and Flack, R. D. (1997b) "Laser Velocimeter Measurement in the Turbine of an Automotive Torque Converter: Part II-Unsteady Measurements", ASME Journal of Turbomachinery, 119, 655-662.

By, R. R. and Lakshminarayana, B. (1995) "Measurement and Analysis of Static Pressure Field in a Torque Converter Turbine", ASME Journal of Fluids Engineering, 117, 473-478.

Dong, Y. (1998) "An Experimental Investigation on Fluid Dynamics of an Automotive Torque Converter", Ph.D Thesis, Department of Aerospace Engineering, The Pennsylvania State University.

Marathe, B. V., Lakshminarayana, B. and Maddock, D. G. (1997) "Experimental Investigation of Steady and Unsteady Flow Field Downstream of Automotive Torque Converter Turbine and Inside the Stator, Part 1: Flow at the Exit of Turbine, Part 2: Unsteady Pressure on the Stator Blade Surface", ASME Journal of Turbomachinery, 119(3), 624-645.

Von Backstrom, T. W. and Lakshminarayana, B. (1996) "Perspective: Fluid Dynamics and Performance of Automotive Torque Converters: An Assessment", ASME Journal of Fluids Engineering, 118, 665-676. 

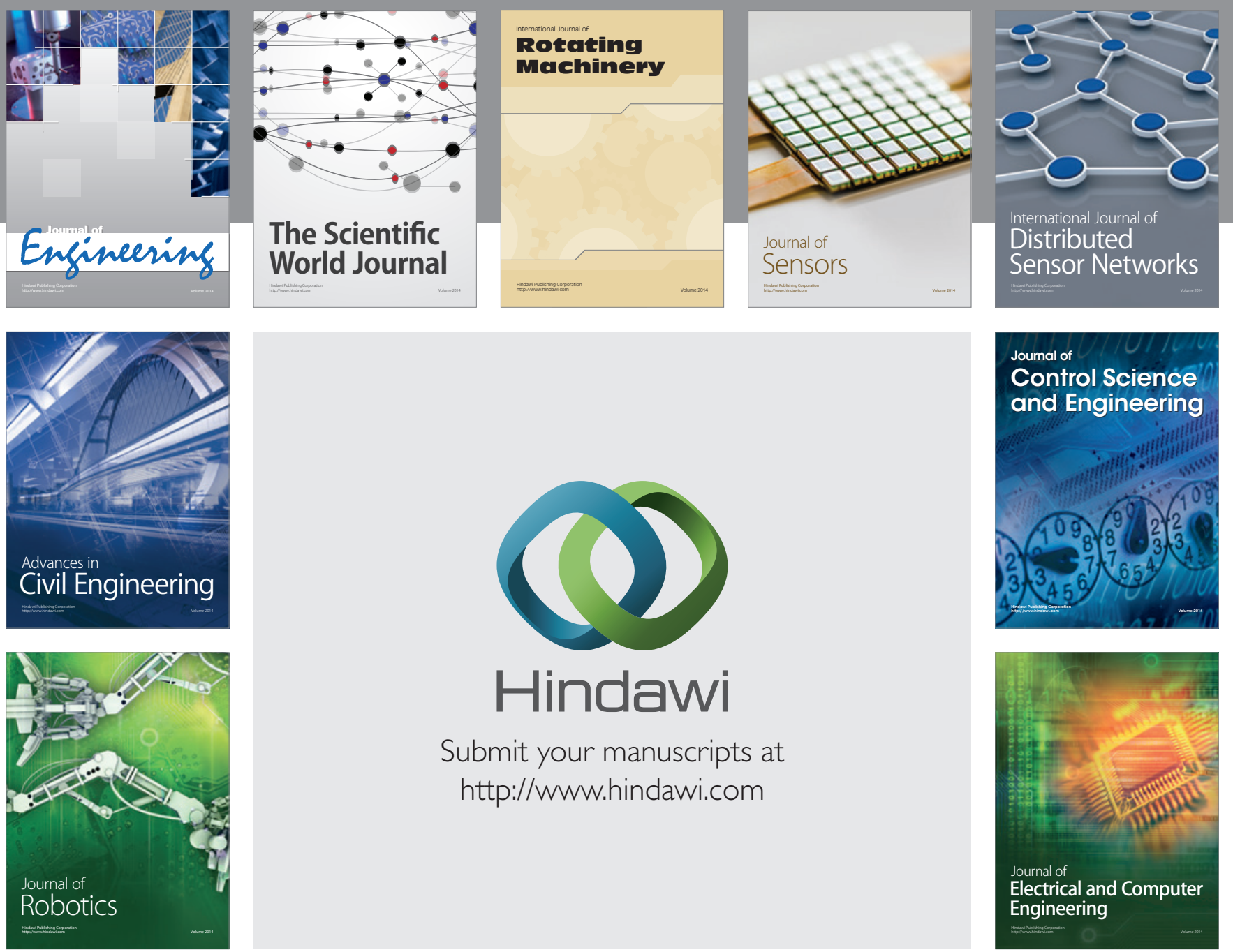

Submit your manuscripts at

http://www.hindawi.com
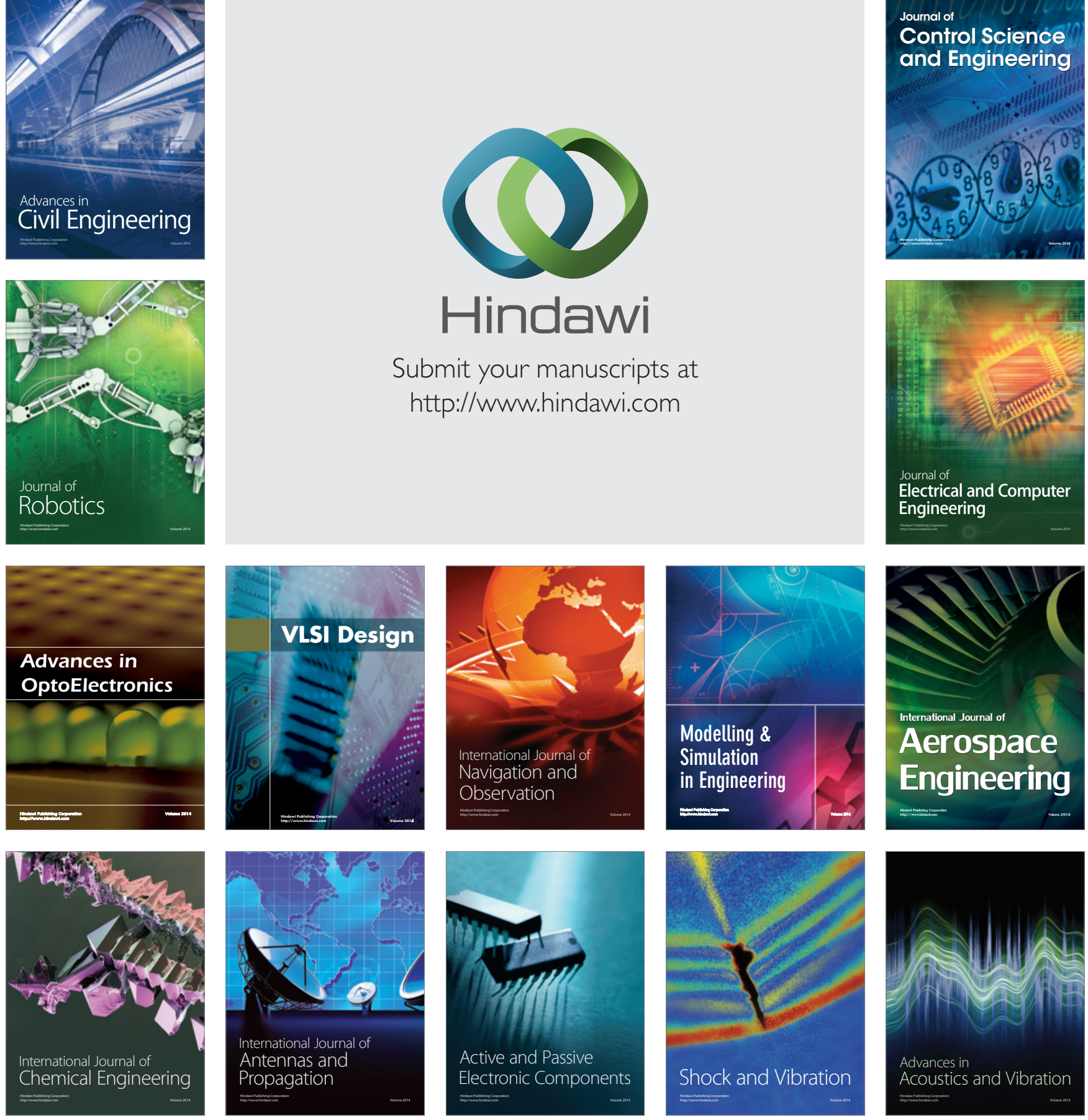